\title{
Entrevistas participativas en diarios digitales: modelos y oportunidades de interactividad
}

\section{Participatory interviews in digital newspapers: models and opportunities for interactivity}

\author{
María-Luisa Sánchez-Calero
}

Cómo citar este artículo:

Sánchez-Calero, María-Luisa (2020). "Entrevistas participativas en diarios digitales: modelos y oportunidades de interactividad". Profesional de la información, v. 29, n. 6, e290626.

https://doi.org/10.3145/epi.2020.nov.26

Artículo recibido el 06-03-2020

Aceptación definitiva: 08-06-2020

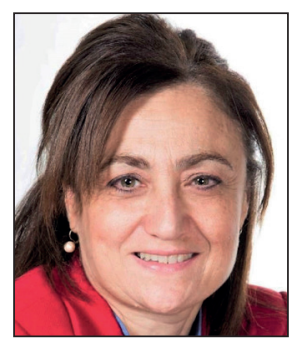

\section{María-Luisa Sánchez-Calero}

https://orcid.org/0000-0002-7929-144X

Universidad Complutense de Madrid

Facultad de Ciencias de la Información

Avda. Complutense, 3

28040 Madrid, España

mlusaca@ucm.es

\section{Resumen}

Se analiza la evolución de los formatos de entrevistas participativas aparecidos desde 2015 a 2020 en las cabeceras digitales de referencia en España (El país, El mundo, $A B C, A S$, El diario.es, El confidencial), y en Facebook live como canal novedoso de distribución de contenidos en redes sociales. Se analizó el contenido de una muestra de más de 300 entrevistas digitales considerando: esquema narrativo entre entrevistado-entrevistador, estilo del nuevo formato en la Red, nivel de participación de sus audiencias, y la interconexión del entrevistado con su público. Se analizaron los niveles de interacción y reacción de las audiencias, y si existe un nuevo storytelling periodístico con diferentes niveles de interacción que busca hacer partícipe a sus audiencias, permitiendo que se sientan parte del diálogo, y que amplíen otras posibilidades de participación en el escenario de la entrevista. El nuevo marco propicia que los cibermedios evolucionen hacia espacios más dialógicos con sus audiencias utilizando un lenguaje textual, gráfico o auditivo que construyan un relato narrativo, que alimente la curiosidad y suscite el interés de participación de su público/lector.

\section{Palabras clave}

Periodismo digital; Ciberperiodismo; Diarios; Prensa digital; Entrevistas participativas; Géneros dialógicos; Storytelling periodístico; Periodismo inmersivo; Interactividad; Audiencias; Participación de las audiencias; Narrativas periodísticas.

\begin{abstract}
The evolution of participatory interview formats that appeared from 2015 to 2020 in the leading digital newspapers in Spain (El país, El mundo, ABC, AS, El diario.es, and El confidencial), as well as Facebook live because it is a new channel for content distribution via social networks, is analyzed. The content of a sample of more than 300 digital interviews was analyzed, considering the narrative scheme between the interviewee and interviewer, the style that the new format acquires on the Internet, the level of audience participation, and the interconnection of the interviewee with the audience/reader. The levels of interaction and reaction of the audiences were analyzed, as well as the possibility of a new type of journalistic storytelling with different levels of interaction that seeks to achieve audience participation, allowing them to feel part of the dialogue, and to expand other possibilities for participation with the interview. This new format encourages online media to evolve towards more dialogic spaces with their audiences using textual, graphic, or auditory language to build a narrative story that fuels curiosity and arouses interest in participation by the audience/reader.
\end{abstract}

\section{Keywords}

Digital journalism; Cyberjournalism; Participatory interviewing; Interviews; Dialogical genres; Journalistic storytelling; Immersive journalism; Cybermedia; Online media; Newspapers; Digital press; Interactivity; Audiences; Audience participation; Journalistic narratives. 


\section{Introducción}

Durante los últimos años, los diarios digitales han evolucionado incorporando fórmulas narrativas y discursivas para facilitar mayor participación de sus audiencias, gracias al avance de las redes y aplicaciones digitales, que posibilitan mayor cercanía, interactividad, inmediatez e hipertextualidad. Se produce una acción-reacción en una audiencia que reclama mayor feedback y otros modos de recepción y consumo de información.

Los discursos y la manera de contar las noticias en los medios digitales y audiovisuales están dando un giro que permite al público la inmersión en la información. Por su parte, los medios estudian el perfil y los hábitos de sus audiencias para ofrecerles contenidos acordes a sus intereses.

Las tecnologías digitales han cambiado no sólo la forma de hacer periodismo sino también el quién, qué, dónde, cuándo e incluso el por qué: se han transformado las seis W del periodismo (Salaverría, 2019). Este progreso tecnológico ha permitido la aparición de fórmulas narrativas, cada vez más frecuentes en la producción periodística, que potencian la capacidad de interactuar y por lo tanto posibilitan no sólo mostrar, sino poder accionar con la información (Domínguez-Martín, 2010).

Los diarios digitales españoles apuestan por la introducción de recursos interactivos y rivalizan por crear nuevos espacios y formatos. En la última década, la entrevista, como género dialógico, ha evolucionado a formatos que presentan diferencias y semejanzas con los tradicionales. Este espacio, denominado de forma diferente según el medio (nosotros lo acotamos con el término de "entrevista interactiva/participativa"), explota diversas funciones interactivas que dan lugar a fórmulas muy elaboradas de presentación de los contenidos. Algunos de los cambios son:

- el nuevo rol de gatekeeper que adopta el periodista para seleccionar y dar cohesión al estilo o formato;

- el proceso e innovación que desarrolla;

- las pautas para la transcripción del diálogo.

\section{Estado de la cuestión}

Antes de abordar las características de estos nuevos modelos dialógicos se aportan unas pinceladas sobre la evolución de la entrevista en los medios de comunicación a lo largo de los siglos XX y XXI. Este género ha gozado siempre de alta reputación y hoy sigue disfrutando de popularidad entre periodistas y audiencias, tanto en los medios tradicionales como en los digitales.

Para Cantavella-Blasco (2007) la mayor ventaja que adquirió la entrevista como género fue la de conseguir un nombre propio. Después de un siglo de ser denominada interviú castellanizó su denominación y pasó de ser considerada un prototipo de reportaje a lograr un status propio. Autores como Martínez-Albertos identificaban la entrevista con la estructura formal del reportaje de citas donde se alternan las palabras textuales del personaje entrevistado con descripciones o narraciones por parte del periodista (Martínez-Albertos, 1983).

La entrevista es y será el arte de saber escuchar (Galán, 2019), y el secreto está en que el personaje perciba que el periodista quiere escucharle. Las habilidades para realizar una buena entrevista dependen del entrevistador y de la moderación que se establezca en el diálogo para dar a conocer los aspectos relevantes del entrevistado. La entrevista persigue una realidad conectada con la actualidad, información con datos y opinión para que el público los entienda.

Es un género periodístico afianzado y sólido, y no ha dejado de explorar fórmulas innovadoras para presentar sus contenidos. Los cibermedios han incorporado elementos de interactividad, hipertextualidad e inmersión que han provocado cambios en su esquema narrativo y en la participación de las audiencias (Mancera-Rueda, 2011). El escenario periodístico actual toma elementos del periodismo inmersivo y la realidad virtual para un storytelling en el que se transforma el relato en una experiencia vivida en el escenario de la noticia.

Las propuestas narrativas inmersivas modifican y potencian el periodismo, siendo su principal desafío ontológico otorgar nuevamente al medio su capacidad mediadora (Cruz; Cevallos; Yépez-Reyes, 2017). Esto aporta una bidireccionalidad en el mensaje y origina cambios del esquema comunicativo de la entrevista en las funciones clásicas de emisor y receptor (Mayoral, 2015). Como consecuencia -según este autor- se gana en flexibilidad y se facilita el diálogo, que es justamente el concepto clave que enmarca a la entrevista.

Desde esta misma perspectiva, López-Hidalgo y Fernández-Barrero (2015) resaltan los beneficios de las entrevistas online justificando que los modelos de participación de audiencias son los que más han evolucionado. Aunque el mayor nivel de interacción y de libertad de participación de las audiencias depende del grado de libertad del que disponga el internauta para intervenir en los contenidos.

La participación también es limitada. Según se explica en una entrevista realizada a Rosa Montero y publicada en la sección de entrevistas de El país semanal,

"en la entrevista, la participación del lector está circunscrita a las normas del medio y las pautas que va marcando el periodista. Pautas que deben ir y van más allá del rol del moderador ya que educa a la audiencia e instruye en su participación y vela por los principios éticos de los textos periodísticos profesionales. Algo que hacen muchos periodistas es querer quedar mejor que el entrevistado. La entrevista no está para eso. Tu misión es que hable el personaje" (Galán, 2019). 
Tanto estudiosos como periodistas resaltan las cualidades de los nuevos modelos, pero advierten de ciertos problemas. Algunos autores expresan su desacuerdo ante la evolución de los formatos digitales ya que -según dicen- han motivado que el género de la entrevista pierda elementos muy consolidados, como la coherencia en el orden temático, el estilo narrativo o el papel de moderador del periodista, entre otros. El perfil del entrevistador conlleva nuevas exigencias profesionales en un contexto marcado por rutinas y tareas más tecnológicas, que es lo que demanda el ecosistema actual (López-García; Rodríguez-Vázquez; Pereira-Fariña, 2017). El nuevo profesional debe conocer los programas, entender los fundamentos de los nuevos modelos y disponer de conocimientos suficientes tanto para el trabajo individual como en equipos interdisciplinares.

También se destacan las ventajas: mayor imprevisibilidad, naturalidad, espontaneidad e inmediatez. Existe un cambio de papeles, una dinámica, composición y esquema narrativo nuevo y se discute si a estos formatos se les puede considerar entrevistas, o si son un género dialógico nuevo. Por ejemplo, aparecen sondeos y encuestas donde el lector vota, se incorporan comentarios donde los públicos expresan sus opiniones, y otras muchas variantes.

Aunque aún es pronto para determinar el éxito o fracaso de los modelos de entrevista participativa -dado los escasos estudios que existen-, son muchos los investigadores, estudiosos y periodistas consultados que reparan en las notables diferencias que se establecen entre ellos (Pulido-Esteban; Sánchez-Calero, 2016). Coinciden en señalar que la fórmula y narrativa que han utilizado en nada o poco tienen que ver con el género de la entrevista convencional y con su estructura, y observan la apuesta que muchos medios digitales están haciendo al integrar los formatos participativos online en la búsqueda del diálogo y la interactuación con sus públicos.

\section{Objeto de estudio}

El objetivo de esta investigación es analizar el grado de participación y los niveles de interacción de las entrevistas participativas digitales publicadas en medios españoles. Se han tenido en cuenta diversos parámetros de análisis con el objeto de comprobar la evolución del género de la entrevista hacia formatos más participativos, analizando aspectos como el grado de interactividad, la inmersión y la participación. El medio de comunicación otorga al lector un poder de participación tanto en la selección de contenidos -interactividad selectiva- como en las posibilidades de expresión y comunicación -interactividad comunicativa-.

Este estudio analiza la estructura, estilo, formato, procedimientos de planificación, producción y distribución, sus posibilidades de interactuar de forma inmediata, y la respuesta de las audiencias a los nuevos formatos. La muestra de análisis consiste en las entrevistas participativas publicadas en los medios digitales El mundo, El país, ABC, El diario.es, AS, El confidencial en sus sitios web, o en espacios como la red social Facebook y con diferentes denominaciones (tabla 2).

Algunos ya han desaparecido por el escaso interés y visibilidad mostrado por el público, pero otros como los Encuentros digitales de El mundo permanecen en activo después de 19 años (la primera edición fue una entrevista a Georgie Dann el 21 de junio de 2000).

Hoy el formato de entrevista online sigue evolucionando en presentación, formato y estilo, como sucede en el periódico El mundo. Sin embargo, la falta de afianzamiento y consolidación en otros medios digitales es también una realidad contrastada y analizada en este artículo.

\section{Metodología}

Para la consecución de los objetivos señalados en torno a la entrevista participativa, y con el objeto de ofrecer un diagnóstico de los nuevos públicos y detectar los retos y oportunidades se hace uso de la siguiente metodología.

Se emplea esencialmente una metodología cualitativa, de análisis crítico, revisión bibliográfica, y recopilación documental en el marco de los medios digitales y en lo referente a los nuevos formatos periodísticos. Para ello se presta atención al impacto de las tecnologías, al desarrollo de nuevos soportes, y a las narrativas que emplean los medios, así como a la interconexión de trabajos científicos y otros trabajos llevados a cabo en proyectos profesionales innovadores relacionados con esta investigación.

Se recurrió a la triangulación metodológica de técnicas cualitativas y cuantitativas (Gaitán-Moya; Piñuel-Raigada, 1998). Se usa el análisis de contenido para registrar de forma sistemática la exploración y el análisis de los modelos aparecidos en diarios digitales de referencia según el EGM en España. Para la muestra de análisis se tomaron diarios digitales que provienen de la prensa impresa como El país, El mundo, ABC y nativos digitales como El diario.es, o El confidencial, añadiendo como ejemplo de información especializada el diario deportivo AS así como Facebook live y YouTube como canales sociales de distribución de contenidos.

En primer lugar se realizaron entrevistas en profundidad a los periodistas responsables o colaboradores de estos espacios (ver sección 7), quienes han facilitado información sobre el proceso de elaboración y en las que se han podido detectar los retos y oportunidades de los modelos. La mayoría de las entrevistas se realizaron de forma presencial y otras vía 
telefónica, entre los meses de febrero y junio de 2019. La elección de los periodistas de la muestra responde a la responsabilidad que estos han tenido o tenían en el desarrollo de estos formatos. Además, el trabajo de campo incluyó técnicas cuantitativas mediante el análisis de contenido de entrevistas participativas escogidas en cada uno de los seis periódicos. Esto supuso visionar unas 300 entrevistas con el objeto de ver su evolución y rescatar de la muestra total las aparecidas en los últimos cinco años (2015-2020). Se seleccionaron entrevistas representativas que tuvieran participación del público, y se realizó un análisis cuantitativo y descriptivo de los formatos y textos.

Para comparar y conocer su evolución se ha utilizado tanto la información de las entrevistas realizadas a los periodistas responsables, como el análisis de las entrevistas participativas publicadas por cada cabecera. Se ha extraído información sobre:

- producción y desarrollo del encuentro;

- promoción;

- denominación del espacio;

- lugar de ejecución;

- elección del entrevistado;

- papel del periodista-entrevistador;

- regularidad, tiempo y duración;

- estructura, redacción y estilo,

- nivel de interacción del público con el entrevistado y

- tipo de discurso.

Los temas tratados en la investigación se muestran en la tabla 1.

Tabla 1. Ejes temáticos planteados en las entrevistas realizadas a los responsables de los espacios dialógicos de los diarios digitales

\begin{tabular}{|l|}
\hline \multicolumn{1}{|c|}{ Ejes temáticos } \\
\hline Fórmulas narrativas que el medio adopta para la participación de sus audiencias. \\
\hline Selección de géneros periodísticos que más se prestan a la participación e interacción. \\
\hline Interacción y visibilidad de las entrevistas con los públicos/lectores y comparación con modelos tradicionales. \\
\hline Origen y precedentes de los nuevos modelos participativos online. \\
\hline Desarrollo, preparación y ejecución de los formatos. \\
\hline Estructura, redacción y composición que establecen en su narrativa y escritura: cuantas preguntas formulan, cuántas contestan, orden en su escritura. \\
\hline Nuevo rol que adoptan el periodista y el entrevistado. \\
\hline Resultados obtenidos mediante la incorporación de este formato. Análisis de sus éxitos y fracasos. \\
\hline
\end{tabular}

Con la combinación metodológica utilizada se pretende localizar un patrón de comportamientos que permita dar respuesta a las siguientes preguntas:

- ¿Cuáles son las ventajas y desventajas respecto al modelo de entrevista tradicional?, y

- ¿¿qué influencia tiene la participación, interactividad, inmersión o hipertextualidad en la creación de un nuevo formato dialógico que interese a sus públicos?

\section{Resultados de la muestra de análisis}

El análisis aborda el estudio de estos formatos dialógicos entre 2015 y 2020. En cada apartado se muestran elementos relevantes y ejemplos destacados.

\subsection{Denominación del espacio}

Las dos denominaciones más utilizadas para este espacio son Encuentro digital y Entrevista digital, aunque también hay otros nombres (tabla 2).

Los periodistas entrevistados del periódico El país aluden a que el medio utilizó diferentes nombres, pero que para ellos es reconocido como el espacio de la entrevista, ya que la conversación que se establece cuenta con un formato clásico de pregunta-respuesta.

https://elpais.com/elpais/entrevistasdigitales.html

https://elpais.com/agr/encuentros_el_pais/a

En $A B C$ la periodista Laura Pintor señala que se les denominaba -ya desaparecieron-Chat o Charla digital por ser una comunicación en tiempo real entre varios usuarios

https://entrevista-digital.abc.es/videochats/chat-con-coti 
En El mundo se sigue utilizando el mismo nombre de Encuentros en una página muy completa y bien programada: https://www.elmundo.es/elmundo/encuentros

En El diario.es, Marilyn Gonzalo Airut, responsable de su implantación, explica que se denomina Pregúntame y la primera fue dirigida a su director Ignacio Escolar.

https://www.eldiario.es/redaccion/Lanzamos-Preguntame-encuentros-digitales-eldiarioes_6_343375696.html

Tabla 2. Denominación del espacio

\begin{tabular}{|l|l|}
\hline \multicolumn{1}{|c|}{ Medios de comunicación digitales } & \multicolumn{1}{c|}{ Denominación de estos espacios } \\
\hline El país & Entrevistas digitales / Encuentros El país \\
\hline El mundo & Encuentros \\
\hline$A B C$ & Entrevista digital/Chat \\
\hline AS & Entrevistas digitales \\
\hline Eldiario.es & Pregúntale \\
\hline El confidencial & Encuentro digital \\
\hline Facebook Live - El mundo & Facebook Live \\
\hline
\end{tabular}

\subsection{Presentación y organización: periodicidad, elección del entrevistado, duración, regularidad, lugar y} papel del periodista

Este tipo de entrevistas no cuentan con una periodicidad fija, pero sí se anuncian con varios días de antelación en la página de inicio de la sección. En El mundo aparece con una breve presentación del entrevistado que actúa de gancho para que los lectores puedan preparar su intervención y formular su pregunta como usuario anónimo. La presentación se acompaña de una foto del entrevistado, el nombre, día y hora de su intervención https://www.elmundo.es/elmundo/encuentros/index.html

En El país se hacía de forma parecida hasta 2016 en el espacio denominado Entrevistas digitales (la última fue al crítico de cine Carlos Bollero). Desde 2014 permanece hasta hoy otro espacio denominado Encuentros El país, dedicado fundamentalmente a escritores. En 2018 se incorporaron novedades como la participación del público en directo, apunta Andrea Nogueira, redactora en Encuentros El país.

https://elpais.com/cultura/2015/11/06/actualidad/1446821655_350810.html

Para la elección del entrevistado en todos los diarios se tiene en cuenta la actualidad periodística, las peticiones de las secciones y las preferencias del público usuario.

Otro denominador común es la participación de los lectores en la entrevista en un tiempo horario fijado. El entrevistado responde a sus lectores, aunque en ocasiones las preguntas son enviadas previamente al periodista y éste las formula en directo. Este tipo de entrevistas permite ver por una webcam al entrevistado mientras responde, aunque existen diferencias entre unos diarios y otros. El número de entrevistas o encuentros que se realizan varía en función del medio y también de la regularidad con la que se retransmiten estos espacios. Una excepción es Encuentros El país donde se invita al público a la entrevista en directo con el invitado: la entrevista la llevan a cabo periodistas del Grupo Prisa y durante los últimos diez minutos el entrevistado responde las preguntas formuladas por el público asistente.

El lugar de realización de las entrevistas participativas suele ser la redacción del diario, donde el entrevistado aparece con el logotipo del periódico. Algunos medios apuestan por el directo en la redacción y se graban para emitir en vídeo parte de la transcripción. En el caso de El país depende de los entrevistados y en ocasiones se les envía un enlace desde el que pueden contestar. La redactora Andrea Nogueiro señala que son entrevistas en directo desde diferentes espacios culturales de Madrid -Teatros Luchana-y el público accede si es suscriptor del periódico o por sorteo en un concurso.

El papel del periodista-entrevistador es fundamental en cualquier entrevista ya que su función es mode-

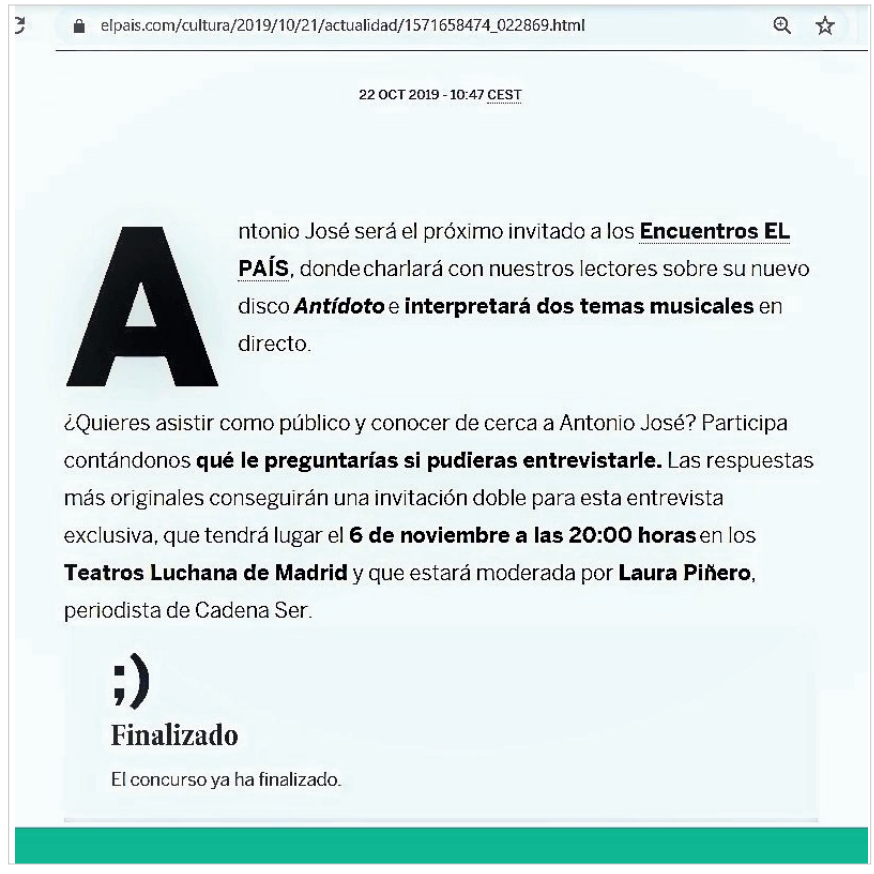

Imagen 1. Presentación del espacio Encuentros El país 
rar, filtrar las preguntas y seleccionar las de mayor interés o desechar las que pueden ser ofensivas. La labor de premoderación conlleva la revisión de preguntas y respuestas antes de su publicación definitiva. Los periodistas redactores o coordinadores del espacio señalan que una de las ventajas del formato es que se gana en participación con los lectores, en variedad y espontaneidad, pero se pierde la intimidad del contexto y la comunicación no verbal entre entrevistado y entrevistador. Otra labor que se descuida es la moderación del periodista para establecer el orden de aparición en el que se transcriben las preguntas y sus respuestas, o la tarea de conectar los temas surgidos durante la charla.

\subsection{Diseño y estilo: redacción, for- mato y estructura}

El carácter de redacción y estilo que debe tener un texto periodístico, en el caso de la entrevista se enmarca en unas pautas comunes que la definen como género dialógico. Sin embargo, la estructura de estos formatos digitales atienden a esquemas diferentes a la entrevista tradicional. En los encuentros y entrevistas digitales la redacción no es homogénea en los medios analizados, y son muchos los casos en que se publican incorrecciones gramaticales, de ortografía y de sintaxis (imagen 2). Esto, según explica la periodista de El mundo Lara Álvarez, es una tendencia muy común, ya que se prima la participación online y se intenta no cambiar nada de la intervención del público.

En El confidencial, cuyos primeros encuentros aparecen en 2015 , se sigue una estructura en la que aparece un membrete en su cabecera que anuncia la sección. Debajo, el titular precedido por el nombre y apellido del entrevistado, con una frase entrecomillada, seleccionada por el periodista. A continuación se muestra una fotografía del invitado con pie de foto y debajo una introducción breve -no en todos- con la presentación y el objeto de la entrevista que le relaciona con la actualidad informativa. https://www.elconfidencial.com/deportes/ rugby/2015-10-21/encuentro-digital-rugbyel-confidencial-rodrigo-contreras_1067357

\section{encuentroseg}
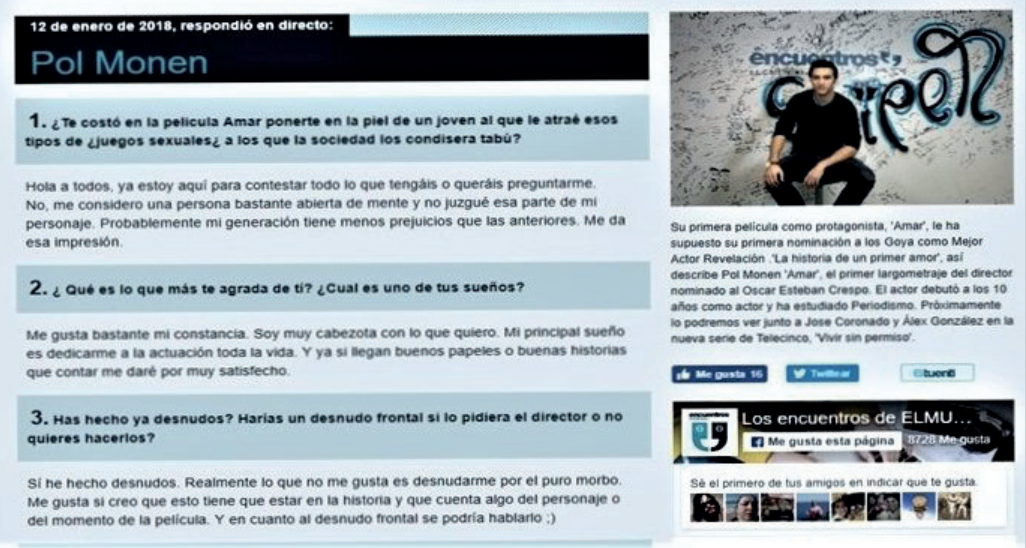

Imagen 2. Muestra la redacción y estilo seguido en las preguntas del espacio Encuentros de El mundo
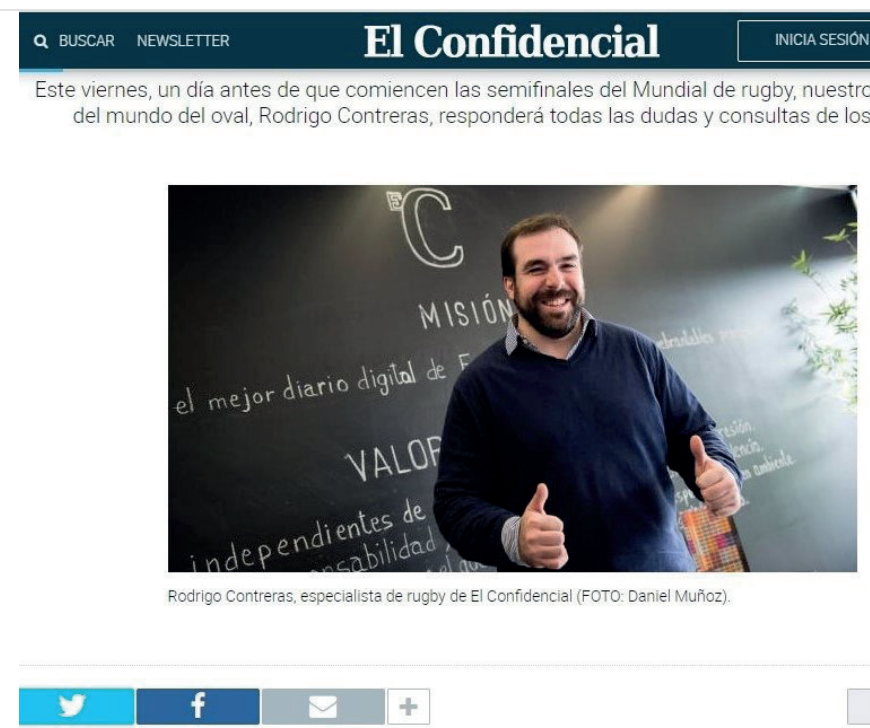

Imagen 3. Presentación del espacio Encuentro Digital de El confidencial

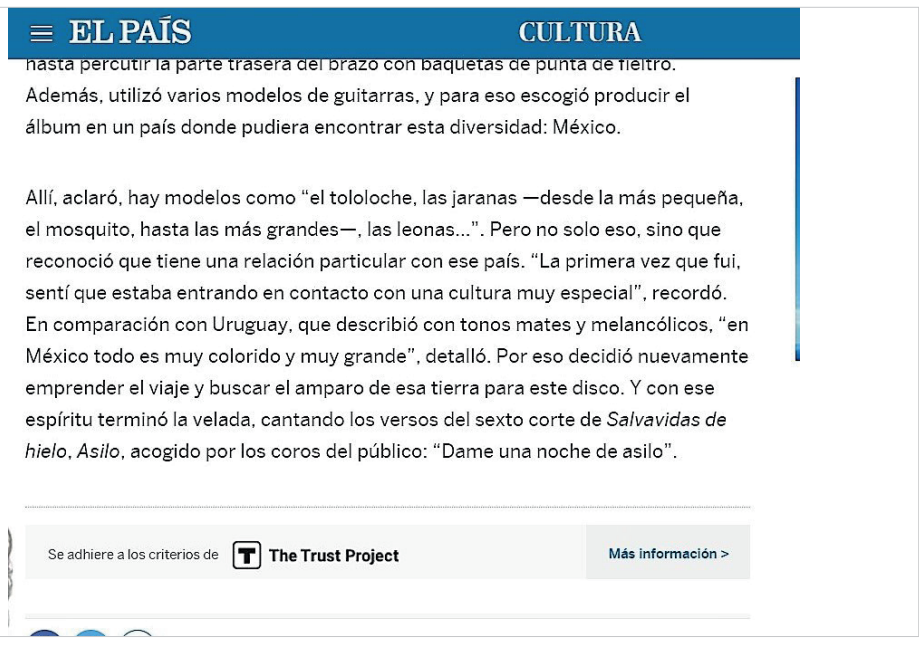

Imagen 4. Redacción del espacio Encuentros El país

También aparece el logotipo del medio donde se realiza la entrevista, para aportar cercanía y actualidad con el lector (imagen 3) y los iconos de las redes sociales del diario, que preceden a las preguntas y respuestas.

En los Encuentros El país -sólo accesible por suscripción- la redactora Andrea Nogueira Calvar comenta que en su formato, el redactor narra lo sucedido en el encuentro (imagen 4). Comenta el lugar del encuentro y la entrevista incluyen- 
do citas con declaraciones del entrevistado. Introduce un ladillo, el titular, un subtítulo y el texto con la inclusión de vídeos de un minuto de duración.

https://elpais.com/cultura/2017/11/29/ actualidad/1511960718_705636.html

En esta investigación se comprueba que la estructura más empleada por estos formatos de entrevistas es la que comienza por la introducción de las preguntas formuladas por los lectores. Emplean tanto un lenguaje formal como coloquial e incluso en muchos casos aparecen los errores gramaticales y ortográficos empleados por el público (imagen 5). También se observa la utilización de varios idiomas en un mismo encuentro y el uso de elementos icónicos en la conversación.

En su estructura se aprecia también la referencia al número total de preguntas formuladas, que puede variar de unas entrevistas a otras. En El mundo aparecen 23 preguntas pero en otros diarios digitales superan las 200. https://www.elmundo.es/elmundo/encuentros/elmundo/2018/03/07/antonio-lobato/index.html

En otros medios como en El confidencial llegan a alcanzar más de 70 preguntas y respuestas https://www.elconfidencial.com/economia/2018-02-14/juan-ramon-rallo-chat-liberal-encuentro-digital-liberalismo_1521717

La extensión depende del interés del entrevistado, aunque suelen durar una hora aproximadamente. Como argumenta la periodista Marilyn Gonzalo Airut, coordinadora del espacio en El diario.es, la selección final realizada por el periodista depende del material del que disponga de cada encuentro.

El diseño es similar en todos los medios analizados: junto a cada pregunta escrita en negrita suele aparecer el número de orden, la fecha y hora de la publicación y el nombre o apodo de quien ha formulado la pregunta. Y a continuación las respuestas a cada una de ellas.

En medios como El país se acompaña de un vídeo de la entrevista de corta duración. A la hora de publicar la transcripción, son los profesionales del medio los encargados de editar y seleccionar las preguntas principales, e incluso pueden agrupar varias en una.

Otro aspecto singular es la despedida (imagen 6) que es uno de los puntos más homogéneos en todos los medios. Aparece la palabra "Despedida" al finalizar la última pregunta redactada, y el entrevistado suele dar las gracias por el interés de los participantes. En algunas ocasiones hace promoción de su libro, película, obra de teatro, etc.

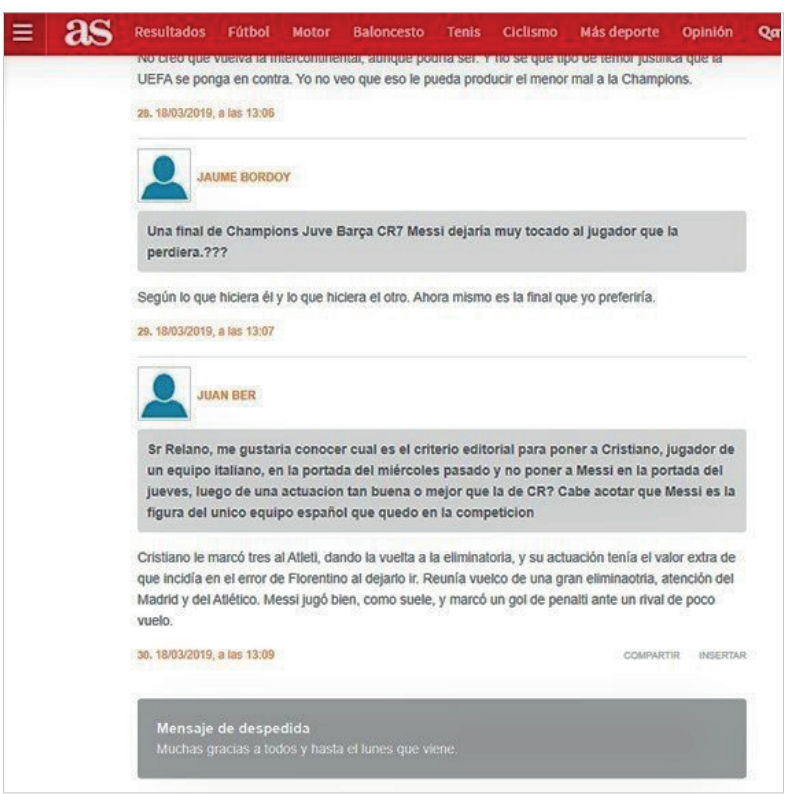

Imagen 6. Ejemplos de despedida de la entrevista en El mundo y AS 


\subsection{Papel del profesional}

El conductor de la entrevista es el periodista, que cobra relevancia cuando cumple con el papel de intermediador entre entrevistado y audiencia. Esa interconexión con el lector se mantiene cuando marca el ritmo de la entrevista, modera las preguntas, filtra, selecciona y auxilia al entrevistado en las respuestas. Aunque aborda otras funciones, una vez finalizada la entrevista revisa los textos, corrige erratas y ortografía, y selecciona el titular procedente de alguna respuesta significativa. Sin embargo, en esta labor se han observado cuestiones negativas como desorden en las preguntas, escasa conexión entre los temas, o poca homogeneidad en los términos de su redacción.

Las entrevistas participativas han tenido una amplia presencia en los medios de comunicación digitales durante la última década y aunque cada vez alcanzan mayor visibilidad y presencia en el conjunto de los contenidos del medio, aún les falta ganar en madurez, como afirman los periodistas consultados.

Si bien se ha consolidado como formato, con una periodicidad, espacio fijo y difusión en las redes sociales, se evidencian carencias en el liderazgo que debería ejercer el periodista en su papel de intermediario entre el entrevistado y la audiencia para consagrar así su labor de gatekeeper.

\subsection{Previsión e innovación de futuro}

La consolidación de la entrevista participativa la ha llevado a adentrarse en nuevos modelos y formatos en las redes sociales. Este es un tema al que alude Jeff Jarvis de la City University of New York en una entrevista publicada en julio de 2019 en el diario El país,

"los periodistas tenemos que ir donde están los lectores y no seguir esperando a que vengan a nosotros" (Pérez-Colomé, 2019).

Si en espacios de los encuentros o entrevistas digitales publicados en los sitios web de los medios ya destacaba como un elemento clave la participación de la audiencia, con la incorporación de la plataforma Facebook Live se han incrementado aún más las posibilidades interactivas.

En los primeros meses de 2016 se lanzó la plataforma Facebook Live en España y los medios no tardaron en sumarse a ella. El país fue el primero en el mes de julio, y El mundo se sumó dos meses más tarde. Facebook Live ha supuesto un antes y un después en los modelos de entrevistas emitidas por los medios, pues ofrece nuevas prestaciones para la realización y distribución de contenidos.

https://www.youtube.com/watch?v=uFfus07dXMI

En Facebook Live se puede informar de la programación, como se aprecia en los encuentros digitales, o ciberencuentros (imagen 7).

En noviembre de 2018 los Encuentros El país introdujeron la fórmula de suscripción para acceder a estos espacios. Se trata de entrevistas muy visibles en Facebook Live en la que los ciudadanos pueden preguntar durante los últimos diez minutos.

https://elpais.com/cultura/2018/11/19/actualidad/1542626978_913480.html

El país y El mundo son los dos diarios digitales españoles que más han apostado por la innovación en el género de la entrevista, y aprovechan las redes sociales para conseguir más interacción entre público y entrevistado. Las diferencias más

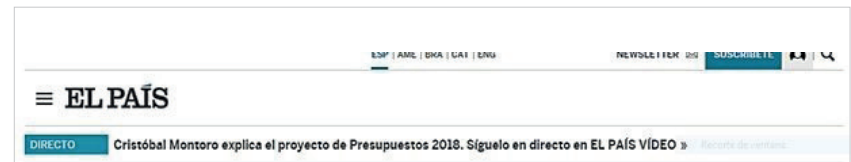

\section{Programación de EL PAÍS en Facebook Live}

Sigue toda la actualidad en nuestra programación especial en directo

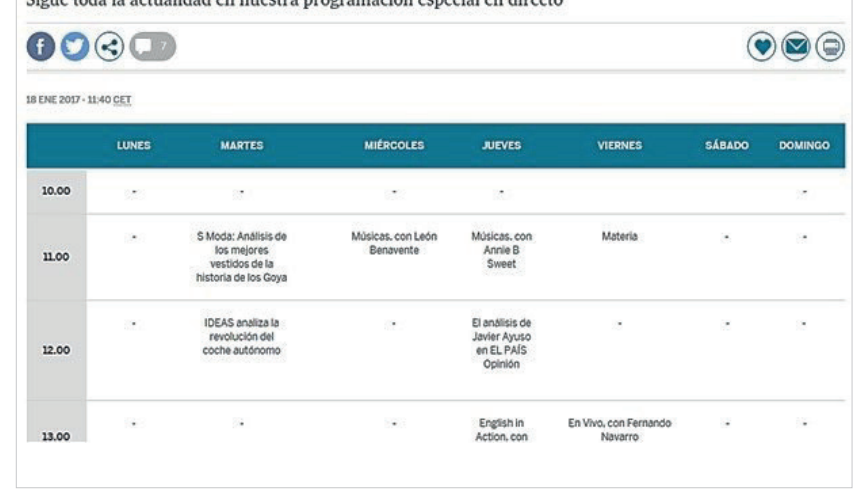

Imagen 7. Ejemplo de programación de Facebook Live de El país

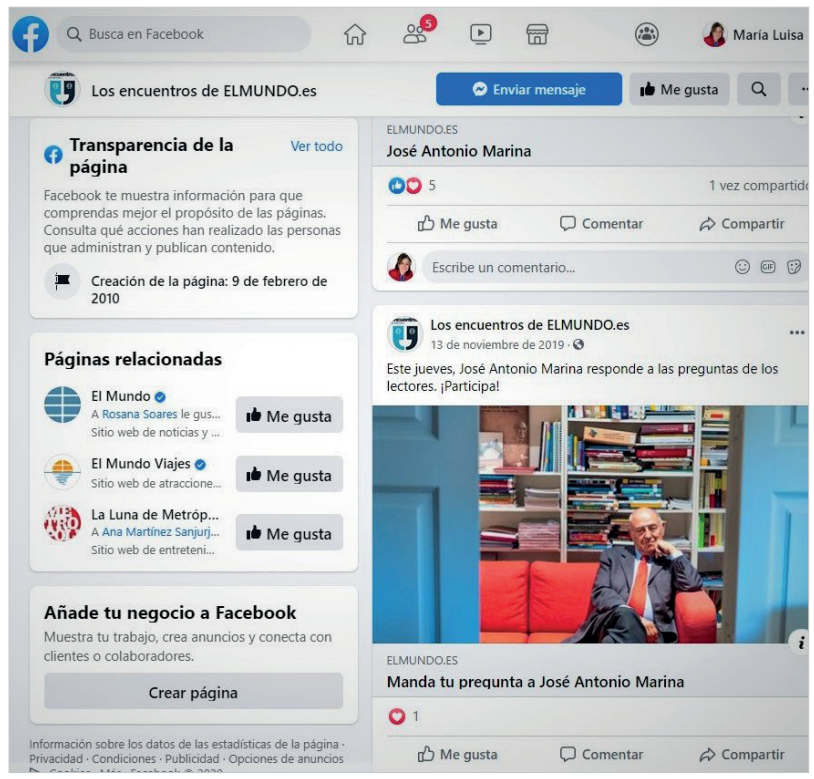

Imagen 8. Encuentros de El mundo en Facebook Live 
notables en los encuentros digitales de ambos diarios son las siguientes:

Los temáticas son muy parecidas en ambos diarios aunque en El país son más usuales los temas políticos, sanitarios, seguidos de culturales, internacionales, medios de comunicación, tecnologías, etc., y en El mundo destacan música, estrenos de cartelera y presentación de obras literarias.

Resulta también Ilamativo que Facebook Live de El país recurra en estos encuentros a entrevistas con periodistas de la propia redacción que son exclusivas periodísticas y permiten a la audiencia preguntar sobre cuestiones de interés.

No existe una periodicidad marcada o un número de encuentros delimitados por día. Este número varía visiblemente de unos meses a otros tanto en El país como en El mundo. La causa se desconoce, pero podría deberse a la falta de consolidación del modelo o a la adaptación de los medios al "mundo sin horarios" de la red de redes. Tampoco tienen establecido un tiempo de duración, aunque normalmente ocupan un tiempo inferior a una hora.

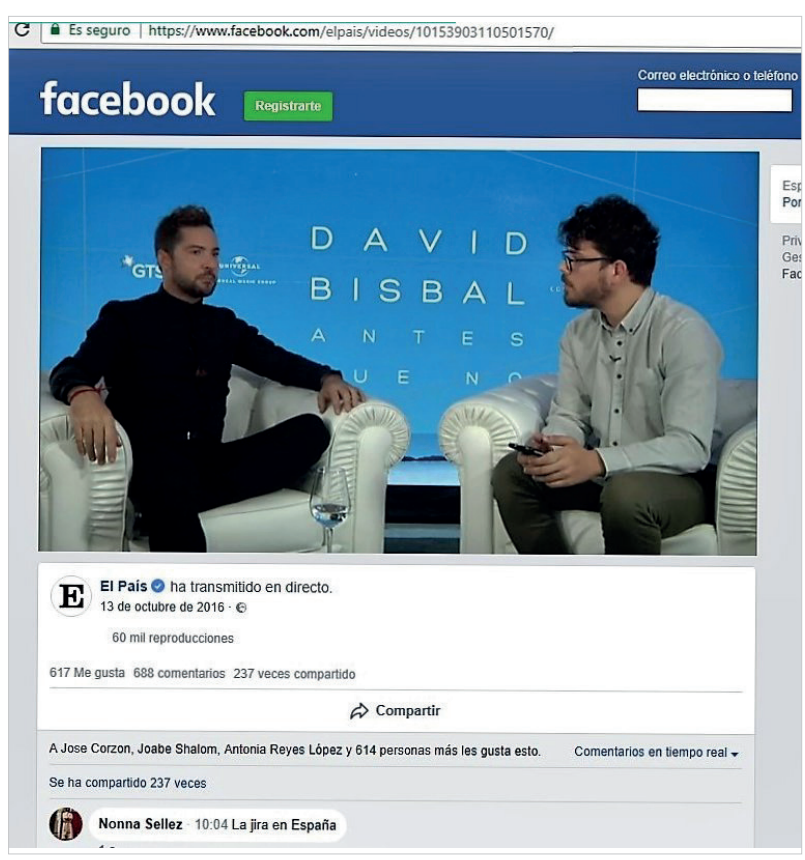

Imagen 9. Encuentros de El país en Facebook Live

Las posibilidades de visualización se incrementan notablemente, ya que otra de las ventajas de la red social es su posibilidad de emitir una alerta a todos los seguidores del perfil indicando que se está realizando un directo. Una vez finalizado el encuentro, el vídeo puede mantenerse publicado en Facebook para que se pueda reproducir más tarde y tantas veces como se desee. El número de visualizaciones de las entrevistas emitidas por Facebook Live dependen del número de seguidores del perfil, lo que justifica que ese número de visualizaciones en El país sea mayor que en El mundo.

Otra de sus particularidades es la interacción con la audiencia, siendo muy similar en El país y en El mundo. No sólo se trasladan comentarios y opiniones, sino que se pueden transmitir impresiones mediante diversos elementos como iconos para valorar el contenido con las opciones "me gusta", "me encanta", "me divierte", "me asombra", "me entristece" y "me enfada". Estas acciones permiten conocer la opinión del público sobre el personaje o sobre el espacio https://www.facebook.com/watch/live/?v=10153903110501570

El usuario en ambos medios puede compartir la entrevista entre sus seguidores en directo. Esto tiene un efecto multiplicador y dinamizador ya que estos seguidores se suman a los del propio medio.

Se permite trasladar las preguntas de las audiencias a la persona que dirige el encuentro para formularla al entrevistado en ambos medios. Ofrecen la oportunidad de seguir los comentarios que se realizan en el espacio e incluso de responderles personalmente.

La asistencia de un moderador es visible en El país y en El mundo, que suele ser un periodista del medio. Asume la función de seleccionar las preguntas de la audiencia y

Muchos medios digitales están integrando los formatos participativos online mantiene el hilo conductor a lo largo de todo el encuen-

tro. Es importante que esté documentado, ya que dirige los ejes de la entrevista en su ejecución. Su labor no es fácil, pues requiere una doble atención para mantener la conexión con los usuarios durante todo el encuentro a través de diferentes dispositivos conectados (imagen 9). Resulta complicado conducir los ejes de la entrevista en estas circunstancias y, por ejemplo, introducir las cuestiones del público en el momento idóneo y sin romper la línea de la conversación.

\section{Conclusiones}

Las hipótesis planteadas han sido verificadas mediante un análisis de contenido extenso y con las declaraciones obtenidas por entrevistas en profundidad a los periodistas que han creado el formato y que en muchos casos siguen trabajando como redactores en los diarios.

Nos encontramos en un nuevo escenario periodístico en el que se prima la creación de espacios más inmersivos y de participación con las audiencias. La entrevista con participación de los lectores es uno de los géneros que apuesta por narrativas dialógicas que faciliten una mayor interactividad, participación e inmersión. El análisis de los diarios digitales El país, El mundo, $A B C, A s$, El diario.es, El confidencial, o en Facebook Live y las declaraciones de los periodistas entrevistados -responsables o creadores de estos espacios de entrevistas- aportan datos tanto sobre los aspectos negativos como los positivos de estos formatos. Entre las conclusiones se pueden destacar las siguientes: 
La entrevista participativa como género dialógico busca la participación e inmersión de los lectores pero tiene problemas de afianzamiento en los actuales medios digitales. Aunque comparte un proceso de ejecución muy similar, se aprecian diferencias notables en su estructura, composición y narrativa respecto de la entrevista tradicional.
Se discute si estos formatos son considerados entrevistas, adquieren una nueva categoría, o son una hibridación que apunta a un género dialógico nuevo

La producción es quizá lo más novedoso en cada medio analizado, ya que aunque es sencilla se diferencia de otros géneros periodísticos. Comienza por la inserción de una cabecera que le permite publicitarse para que los interesados puedan ir enviando sus preguntas. El día de la entrevista -normalmente en directo- se inicia con la llegada del entrevistado a la redacción del diario y después de una corta presentación van apareciendo las preguntas y respuestas en la pantalla.

Posteriormente se redactan los textos, aunque destaca la poca elaboración, selección y corrección que existe tanto en preguntas como en respuestas. Sus responsables lo justifican porque quieren que la participación del público sea original y por ello prácticamente no modifican sus preguntas. Además estas suelen aparecer sin orden en los temas planteados y a veces con faltas de ortografía o erratas. Asimismo, las respuestas en muchos casos no amplían, explican ni aclaran.

La duración es de unos 60 minutos, pero la extensión depende del interés que cause el entrevistado, de la participación del público en el encuentro y de la selección final que pueda realizar el periodista. Se ha observado que el número de preguntas y respuestas es muy elevado en la mayor parte de la muestra con la excepción de los diarios El país y El mundo.

El papel del periodista-entrevistador tiene que perfilarse más ya que su papel es conducir y fomentar la participación y en estos modelos no se aprecian sus funciones. Falta contar con una participación mucho más activa por parte de los profesionales garantizando su papel de intermediarios entre el entrevistado y la audiencia para consagrar su labor de gatekeeper.

Para consolidarse como un nuevo modelo de entrevista periodística y contar con una inmersión, participación e interactividad mucho más activa por parte de los profesionales y del público, se hace necesario potenciar su visibilidad en el conjunto de los contenidos del medio y reforzar su presencia e identidad como género periodístico autónomo en el que los lectores asumen el papel de preguntar.

En el estudio se constatan también las nuevas prestaciones que ofrece la red social Facebook Live para la entrevista en cuanto a la realización, la distribución de contenidos y mayores posibilidades interactivas.

Se aprecia asimismo que en los últimos años la entrevista participativa ha conocido un cierto declive ante la falta de afianzamiento y consolidación del modelo. Aunque es reconocida la apuesta de muchos medios digitales por estos formatos participativos online, se hace necesario buscar fórmulas nuevas o alternativas si realmente quiere llegar a ser considerada como género propio de entrevista, y no como un simple cuestionario de modelo alternativo online.

Este trabajo puede servir de base a otras investigaciones futuras sobre el interés por parte del público hacia opciones informativas cada vez más interactivas y participativas. También puede ser de punto de partida para estudiar y comparar el nivel de participación en los diversos géneros periodísticos de los diarios digitales.

\section{Periodistas entrevistados}

- Lara Álvarez, periodista responsable de La otra crónica en EI mundo;

- Marilyn Gonzalo Airut, responsable de la implantación de Pregúntame en Eldiario.es;

- Laura Pintor, en $A B C$;

- Andrea Nogueira, redactora en Encuentros El país;

- Darío Puntero, en AS.

\section{Bibliografía}

Cantavella-Blasco, Juan (2007). Manual de la entrevista periodística. Madrid: Editorial Universitas, S. A. ISBN: 97884 79912062

Cruz, Jorge; Cevallos, Patricio; Yépez-Reyes, Verónica (2018). “Desafíos del periodismo inmersivo”. En: López-Golán, Mónica; Campos-Freire, Francisco; López-López, Paulo C.; Rivas-Echeverría, Francklin (eds.). La comunicación en la nueva sociedad digital. Coeditado por Pontificia Universidad Católica del Ecuador, pp. 65-78. ISBN: 9978773499

Domínguez-Martín, Eva (2015). “Periodismo inmersivo o cómo la realidad virtual y el videojuego influyen en la interfaz e interactividad del relato de actualidad". El profesional de la información, v. 24, n. 4, pp. 413-423.

https://doi.org/10.3145/epi.2015.jul.08 
Gaitán-Moya, Juan-Antonio; Piñuel-Raigada, José-Luis (1998). Técnicas de investigación en comunicación social: elaboración y registro de datos. Madrid: Síntesis. ISBN: 9788477386049

Galán, Lola (2019). "Rosa Montero: 'Muchos periodistas, y me parece destrozar una entrevista, quieren quedar mejor que el entrevistado'". El país, 26 marzo.

https://elpais.com/elpais/2019/03/18/eps/1552907536_482510.html

López-García, Xosé; Rodríguez-Vázquez, Ana-Isabel; Pereira-Fariña, Xosé (2017) “Competencias tecnológicas y nuevos perfiles profesionales: desafíos del periodismo actual". Comunicar, n. 53, pp. 81-90.

https://doi.org/10.3916/C53-2017-08

López-Hidalgo, Antonio; Fernández-Barrero, Ángeles (2015). “La entrevista de los lectores: en la convergencia de medios y audiencias activas. Posibilidades y retos para la participación del lector". Estudios sobre el mensaje periodístico, v. 21, n. 1, pp. 353-367.

https://doi.org/10.5209/rev_ESMP.2015.v21.n1.49099

Mancera-Rueda, Ana (2011). ¿Cómo se "habla" en los cibermedios? El español coloquial en el periodismo digital. Berna: Peter Lang A.G. ISBN: 9783034310703

Martínez-Albertos, José-Luis (1983 [1992]). Curso general de redacción periodística. Periodismo en prensa, radio, televisión y cine. Lenguaje, estilo y géneros periodísticos. Barcelona: Mitre. ISBN: 9788486153038

Mayoral, Javier (2015). Redacción periodística. Medios, géneros y formatos. Madrid: Editorial Síntesis. ISBN: 978 8499588728

Pérez-Colomé, Jordi (2019). “Jeff Jarvis. Profesor y consultor de medios: 'No he sido bastante radical al imaginar el futuro del periodismo"'. El país, 12 julio.

https://elpais.com/tecnologia/2019/07/12/actualidad/1562885709_674849.html

Pulido-Esteban, Felipe; Sánchez-Calero, María-Luisa (2016). "El nuevo concepto de entrevista periodística: la entrevista participativa". En: I Congreso internacional comunicación y pensamiento. Comunicracia y desarrollo social, pp 35,56. ISBN: 9788494524325

https://idus.us.es/xmlui/handle/11441/50421

Pulido-Esteban, Felipe; Sánchez-Calero, María-Luisa (2017). “Facebook Live: un nuevo canal para la entrevista”. En: González-Alba, José-Antonio (coord.). Tendencias e innovación en la empresa periodística. Sevilla: Egregius Ediciones, pp. 85-102. ISBN 9788494697852

Salaverría, Ramón (2019). “Digital journalism: 25 years of research. Review article". El profesional de la información, v. 28, n. 1, e280101.

https://doi.org/10.3145/epi.2019.ene.01

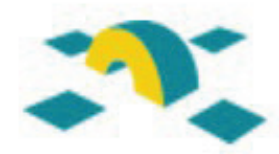

$\nsubseteq|R| S$

\section{COMUNICACIÓN}

\section{https://www.rediris.es/list/info/comunicacion.htm/}

Comunicación es una lista de distribución en castellano para debatir y estar al día sobre teoría de la comunicación, comunicación política, comunicación industrial, relaciones públicas, comunicación audiovisual y multimedia, radio y televisión, cinematografía, periodismo, periodismo de datos, divulgación de la ciencia, medios y cibermedios, redes sociales... y todos los aspectos relacionados con la COMUNICACIÓN.

Empezó a funcionar en enero de 2017 y está alojada en el servicio de listas de RedIRIS, desde donde es posible consultar sus archivos:

https://listserv.rediris.es/cgi-bin/wa?A0=COMUNICACION

La lista cuenta con 2 moderadores que permanentemente filtran los mensajes para evitar spam, mensajes inapropiados, anuncios, mensajes repetidos, etc.:

Isabel Olea (EPI, León)

Tomàs Baiget (EPI, Barcelona)

Puedes suscribirte a Comunicación en:

https://listserv.rediris.es/cgi-bin/wa?SUBED1=COMUNICACION\&A=1 